\title{
Spectroscopy of three strongly coupled flux qubits
}

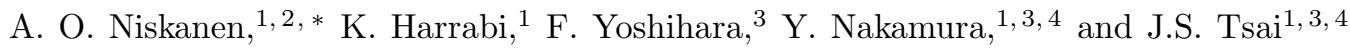 \\ ${ }^{1}$ CREST-JST, Kawaguchi, Saitama 332-0012,Japan \\ ${ }^{2}$ VTT Information Technology, Microsensing, POB 1207, 02044 VTT, Finland \\ ${ }^{3}$ The Institute of Physical and Chemical Research (RIKEN), Wako, Saitama 351-0198, Japan \\ ${ }^{4}$ NEC Fundamental Research Laboratories, Tsukuba, Ibaraki 305-8501, Japan
}

(Dated: May 26, 2018)

\begin{abstract}
We have carried out spectroscopic measurements of a system of three strongly coupled fourjunction flux qubits. The samples studied cover a wide range of parameters with the coupling energy between neighboring qubits varying between $0.75 \mathrm{GHz}$ and $6.05 \mathrm{GHz}$. The observed complicated spectra agree well with eight-level theory. The experiments are relevant for the realization of a tunable coupling between qubits.
\end{abstract}

PACS numbers: 03.67.Lx,85.25.Cp,74.50.+r

The potential realization of a full scale quantum computer requires the ability of coupling multiple qubits together preferably so that the coupling can be turned on and off at will. In the context of Josephson junction qubits there is a number of promising theoretical suggestions ${ }^{1.2 .3}$ as well as already several experiments with coupled qubits 4.5.6.7.8.9.10.11. In order for a quantum computer to be truly scalable it must be possible to couple many qubits together without degrading the coherence time severely. Tunable coupling has not been so far demonstrated in an experiment where the coherence time would be equally good as in the case of so-called optimally biased qubits 12.13 .14 .15 . A problem common with many coupling methods is that in order to realize a twoqubit gate the biases need to be switched away from the region in the parameter space where the decoherence is minimal. In other words, while the coupling between two (or more) qubits is strengthened, the coupling between the qubits and the environment is, in many cases, also strengthened.

This paper describes spectroscopic experiments on three strongly coupled flux qubits, which is relevant for instance for the scheme suggested in Ref. 16. In that scheme the parametric coupling of two detuned optimally biased flux qubits is realized through the microwave modulation of their tunable mutual inductance realized using a third qubit. Thus, the system we study is a set of three antiferromagnetically coupled flux qubits. When the flux threading the qubits is near half flux quantum (modulo flux quantum $\Phi_{0}$ ) the system of three qubits is reasonably well described by the Hamiltonian

$$
H=-\frac{1}{2} \sum_{j=1}^{3}\left(\Delta_{j} \sigma_{x}^{j}+\epsilon_{j} \sigma_{z}^{j}\right)+\sum_{k=1}^{3} \sum_{l=k+1}^{3} J_{k l} \sigma_{z}^{k} \sigma_{z}^{l},
$$

where $\epsilon_{j}=2 I_{\mathrm{p} j}\left(\Phi_{j}-\Phi_{0} / 2\right)$ is the energy bias of qubit $j$ controllable through the flux $\Phi_{j}$ threading the qubit loop. Near the half-flux-quantum point each qubit experiences a double well potential and the tunneling energy through the potential barrier separating the wells is $\Delta_{j}$. The wells correspond to currents of magnitude $I_{\mathrm{p} j}$ circulating in opposite directions along the loop and

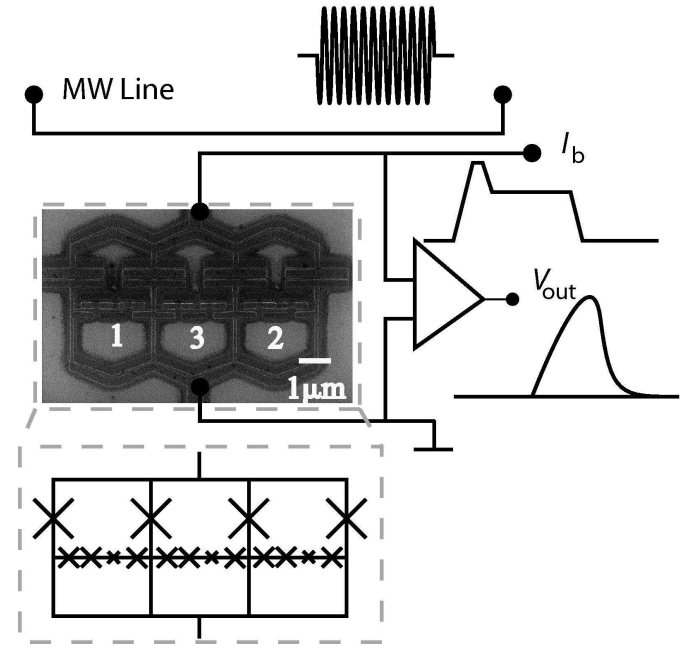

FIG. 1: SEM image of the sample and the principle of the measurement.

the above Hamiltonian is actually written in this circulating current basis. The antiferromagnetic interaction between the qubits $k$ and $l$ is characterized by the coupling strength $J_{k l}=M_{k l} I_{\mathrm{p} k} I_{\mathrm{p} l}$ where $M_{k l}$ is the mutual inductance.

A sample realizing such a system is shown in Fig. 1] The interesting part of the sample consists of three fourJosephson-junction flux qubits, similar to those studied individually in Refs. 13 and 15, coupled together by sharing one edge of the superconducting loop. The qubits 1 and 2 are thus expected to be weakly coupled due to small geometric inductance whereas each of them is expected to be strongly coupled to the qubit 3 through kinetic inductance. Each qubit has one smaller Josephson junction whose area is about $\alpha \approx 0.5$ times smaller than that of the larger junctions having areas of about $200 \mathrm{~nm}$ by 400 $\mathrm{nm}$. The qubits are coupled to a four-junction readout SQUID consisting of the three larger loops. The SQUID can be seen as a dc SQUID whose junctions are replaced by dc SQUIDs. This design was chosen in order to re- 
duce the influence of the inevitable noise in the bias current. The coupling of the qubits to the SQUID is also through kinetic inductance. Since the different states of the qubits correspond to different magnetic field configurations in the SQUID loops we expect a finite population of the excited states to result in a small but detectable change of the switching current of the SQUID.

The samples used in this study were fabricated out of $\mathrm{Al}$ using standard shadow evaporation through a $\mathrm{Ge}$ enforced mask patterned with e-beam lithography. The designs of samples $\mathrm{A}, \mathrm{B}$ and $\mathrm{C}$ were slightly different. In sample A we used an on-chip capacitor to shunt the SQUID $13,15,18$. In samples B and C this capacitor was removed in order to clean up the measurable spectrum from resonances such as LC resonances and the plasma resonance. An additional benefit of removing the large superconducting shunt capacitor was improved flux stability. Both designs had no on-chip bias resistors but instead we used an on-chip LC filter consisting of a long superconducting line $\frac{17}{7}$ and a superconducting capacitor. The two plates of the Al parallel plate capacitor were defined in a separate e-beam lithography step and the insulator was formed by heavily oxidizing the bottom layer. The estimated cutoff of the fliter was $100-200 \mathrm{MHz}$. In sample A the inductor was about $15 \mathrm{~mm}$ long and 600 $\mathrm{nm}$ wide resulting in a transmission line resonance around $10 \mathrm{GHz}$. The shorter line of samples B and C $(3.2 \mathrm{~mm})$ gives a resonance around $20 \mathrm{GHz}$. The oxidation of the junction was done using a mixture of $\mathrm{O}_{2}(10 \%)$ and $\mathrm{Ar}$ (90\%) between the depositions of the $20 \mathrm{~nm}$ and $30 \mathrm{~nm}$ $\mathrm{Al}$ layers. For the microwave line and bonding pads we used evaporated gold film defined by optical lithography. Part of the microwave line also had an Al layer. The measurements were carried out in a dilution refrigerator with a base temperature around $20 \mathrm{mK}$. The fridge had a triple $\mu$-metal magnetic shield.

The principle of the measurement 18 is shown in Fig. [1 First a microwave pulse of typically $5 \mu$ s duration is applied to the microwave line on chip. Then immediately after this a current pulse of about $5-10 \mathrm{~ns}$ is applied to the SQUID. The height is chosen so that the SQUID switches about every second time. The current pulse is followed by a trailing plateau whose height is about $70 \%$ of the switching current. The application of current through the SQUID changes the magnetic fluxes experienced by the qubits and this shift is assumed to be adiabatic. The purpose of the plateau is to maintain the SQUID in the voltage state if and only if the SQUID switched. By repeating each measurement typically $10^{4}$ times and counting the relatively slow voltage pulses (few $\mu$ s) we can deduce the switching probability $P_{\mathrm{sw}}$ under particular circumstances. To supply the flux bias to the qubits we used an external coil capable of inducing about 20 Gs. To carry out spectroscopy on the qubits we first located them in the flux space. The basic measurement was then carried out by sweeping the microwave frequency and magnetic field and repeating the above mentioned measurement scheme. At each flux
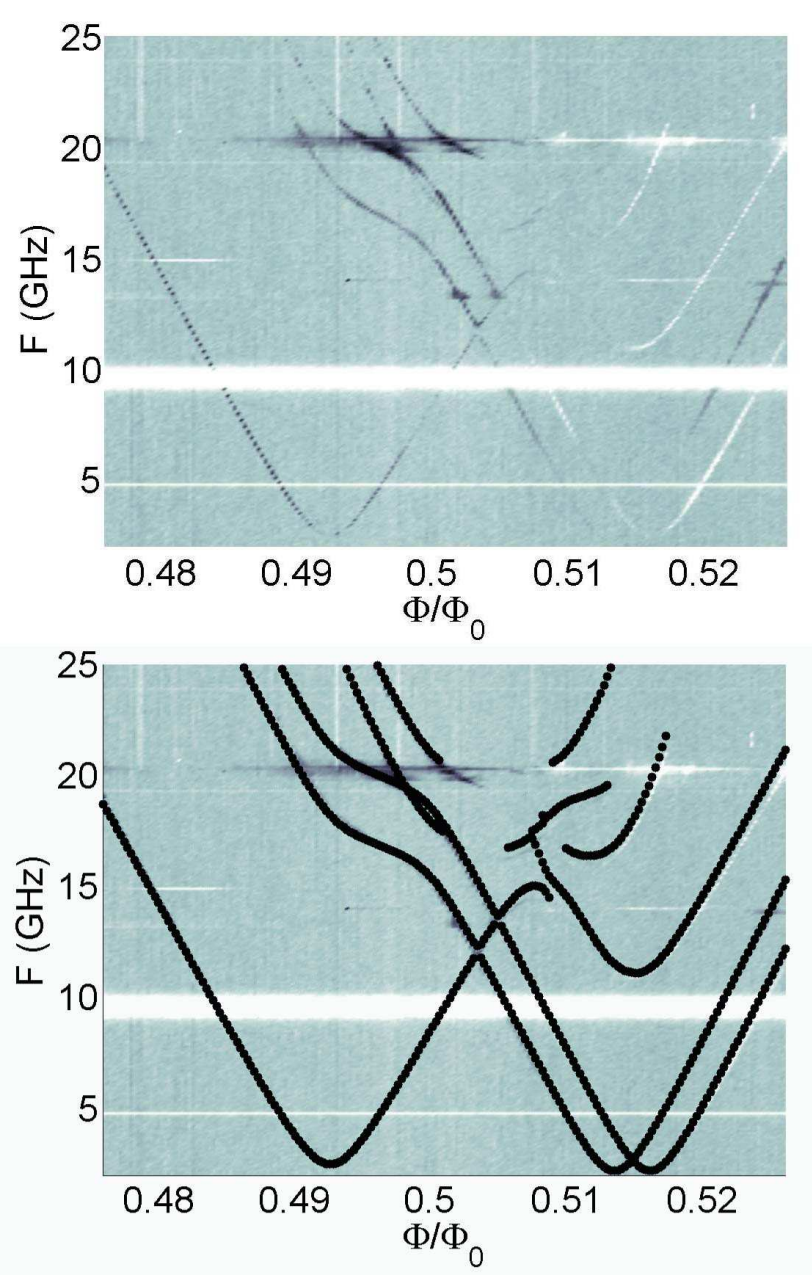

FIG. 2: Spectroscopy of sample A. Top panel is an intensity plot of $P_{\mathrm{sw}}$ while the bottom panel includes the numerically calculated spectrum. Black dots denote excitation energies from the ground state $|0\rangle$ for the states $|\Psi\rangle$ with $\left|\left\langle 0\left|\sum_{j=1}^{3} \sigma_{z}^{j}\right| \Psi\right\rangle\right|^{2} \geq 0.002$. In the calculation we used $\Delta_{1} / h=2.2 \mathrm{GHz}, \Delta_{2} / h=2.2 \mathrm{GHz}, \Delta_{3} / h=2.5 \mathrm{GHz}$, $J_{13} / h=J_{23} / h=2.05 \mathrm{GHz}, J_{12}=0 \mathrm{GHz}, \Delta \Phi_{1} / \Phi_{0}=$ $-0.0128, \Delta \Phi_{2} / \Phi_{0}=-0.0102, \tilde{I}_{\mathrm{p} 1}=194 \mathrm{nA}, \tilde{I}_{\mathrm{p} 2}=194 \mathrm{nA}$, $\tilde{I}_{\mathrm{p} 3}=179 \mathrm{nA}$. The lines shared by the qubits have the dimensions of $1.35 \mu \mathrm{m} \times 30 \mathrm{~nm} \times 160 \mathrm{~nm}$. Qubits 1 and 2 have $\alpha=0.5$ while qubit 3 has $\alpha=0.475$. Oxidation was done with 25 mTorr for 5 min. Switching measurement yields $I_{\mathrm{c}, \max }^{\mathrm{SQUID}} \approx 8.8 \mu \mathrm{A}$.

point the height of the current pulse was adjusted to get roughly the same $P_{\mathrm{sw}}$ in the absence of the microwave. The qubits (as well as other resonances) cause deviations from this probability enabling the characterization of the spectrum of the three-qubit system.

Figure 2 is an example of such a spectroscopic measurement on sample A. The horizontal lines are resonances due to the presence of on-chip capacitors. The strong resonance around $10 \mathrm{GHz}$ is most likely a half-wavelength resonance in the long bias line. However, the qubits are clearly visible and form a rich spectrum. The top panel 

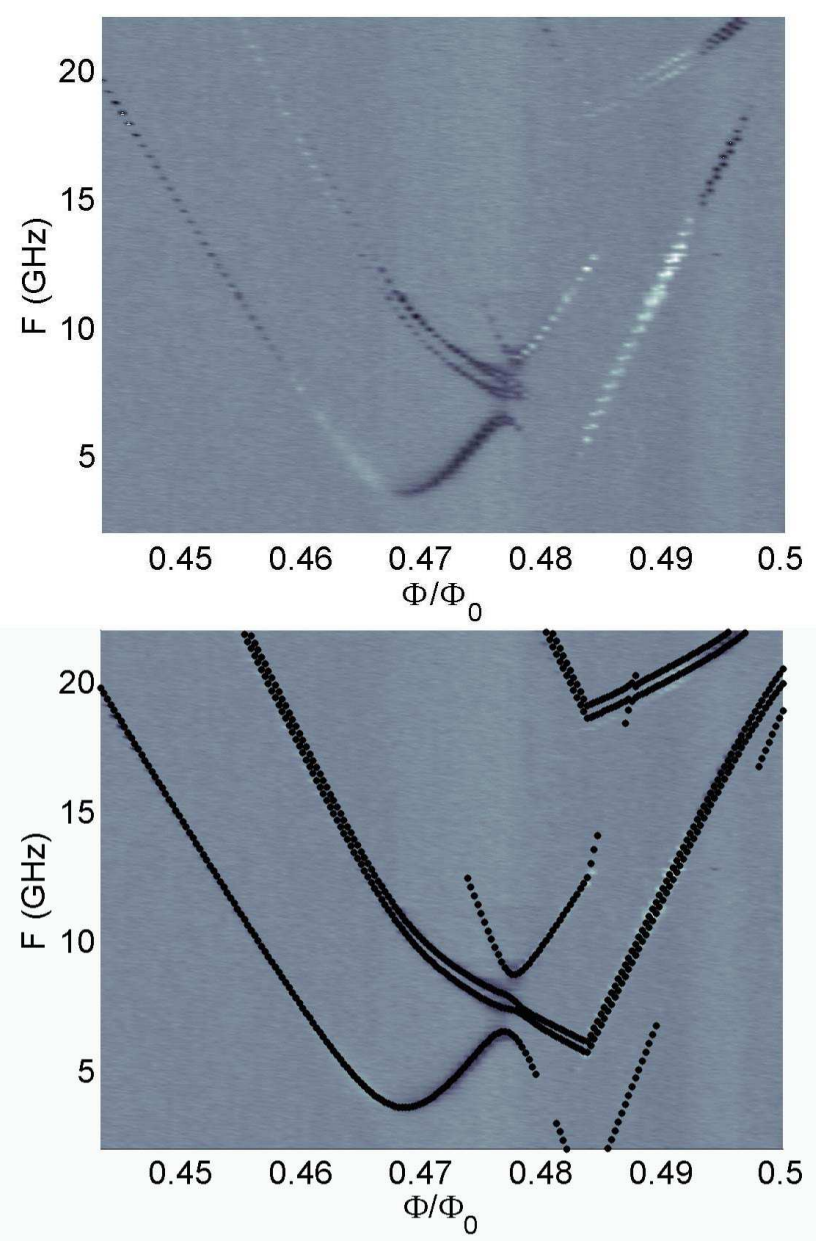

FIG. 3: Spectroscopy of sample B. The parameters used in the calculation are $\Delta_{1} / h=1.3 \mathrm{GHz}, \Delta_{2} / h=1.0 \mathrm{GHz}$, $\Delta_{3} / h=3.6 \mathrm{GHz}, J_{13} / h=J_{23} / h=6.05 \mathrm{GHz}, J_{12}=0$ $\mathrm{GHz}, \Delta \Phi_{1} / \Phi_{0}=-0.0096, \Delta \Phi_{2} / \Phi_{0}=-0.010, \tilde{I}_{\mathrm{p} 1}=156 \mathrm{nA}$, $\tilde{I}_{\mathrm{p} 2}=156 \mathrm{nA}, \tilde{I}_{\mathrm{p} 3}=124 \mathrm{nA}$. We only show levels for which $\left|\left\langle 0\left|\sum_{j=1}^{3} \sigma_{z}^{j}\right| \Psi\right\rangle\right|^{2} \geq 0.0001$. The lines shared by the qubits have the dimensions of $1.35 \mu \mathrm{m} \times 20 \mathrm{~nm} \times 100 \mathrm{~nm}$. Qubits 1 and 2 have $\alpha=0.5$ while qubit 3 has $\alpha=0.45$. Oxidation was done with 25 mTorr for $10 \mathrm{~min}$. We get $I_{\mathrm{c}, \max }^{\mathrm{SQUID}} \approx 20.2 \mu \mathrm{A}$.

shows the measured spectrum only while in the bottom panel a theoretical calculation of the spectrum is shown on top of the measured spectrum. The calculation is simply done numerically using Eq. [ by finding the excited state eigenvalues and subtracting the ground state eigenvalue from them. It is worthwhile stressing that the theoretical part is not based on a fitting procedure such that there may be considerable amount of error in all the parameters, but the agreement does seem at least qualitatively very good. The persistent currents denoted by $\tilde{I}_{\mathrm{p} k}$ in the figure captions are the actual values used in the computation and do not account for the shielding effect. The actual persistent currents are expected to be significantly larger and also the mutual inductance is expected to be smaller than what it seems consider-
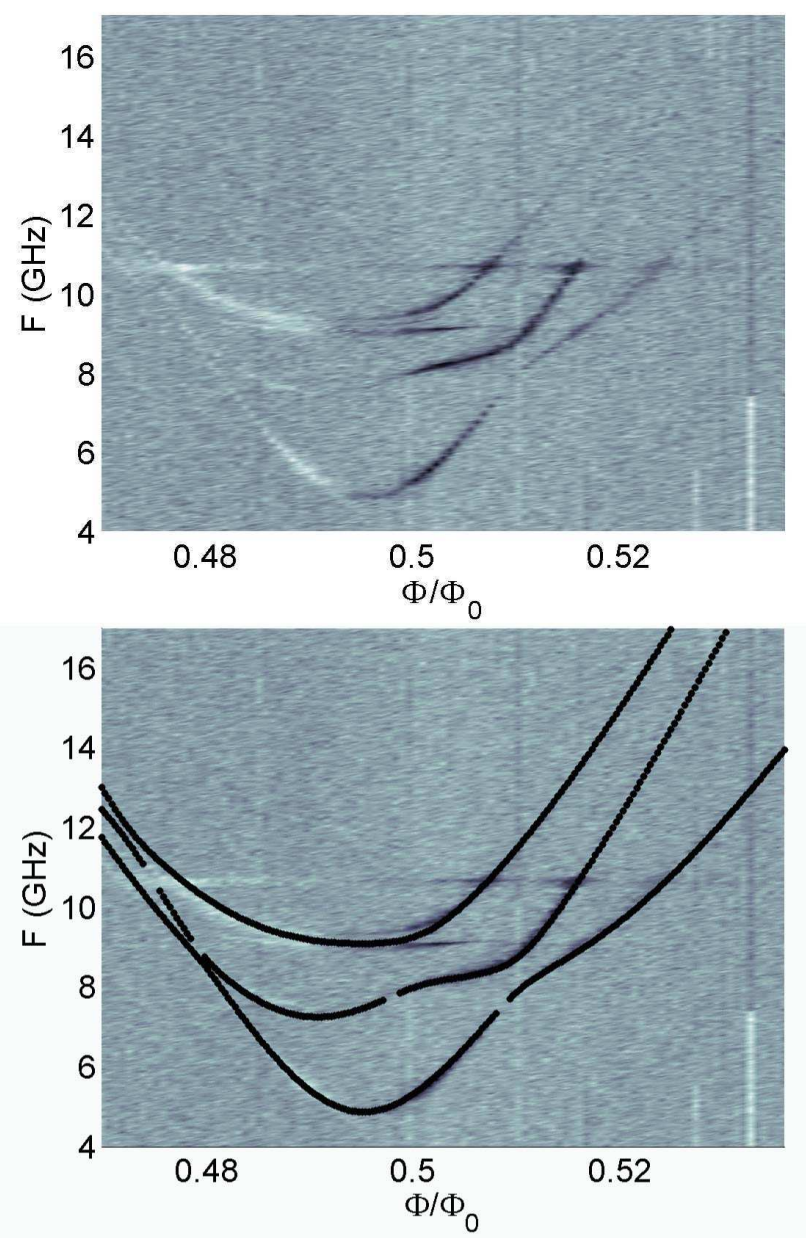

FIG. 4: Spectroscopy of sample C. In the calculation we used $\Delta_{1} / h=5.0 \mathrm{GHz}, \Delta_{2} / h=7.5 \mathrm{GHz}, \Delta_{3} / h=8.2 \mathrm{GHz}$, $J_{13} / h=0.79 \mathrm{GHz}, J_{13} / h=0.755 \mathrm{GHz}, J_{12}=0 \mathrm{GHz}$, $\Delta \Phi_{1} / \Phi_{0}=-0.0041, \Delta \Phi_{2} / \Phi_{0}=-0.0087, \tilde{I}_{\mathrm{p} 1}=81 \mathrm{nA}, \tilde{I}_{\mathrm{p} 2}=$ $78 \mathrm{nA}, \tilde{I}_{\mathrm{p} 3}=64 \mathrm{nA}$. Levels with $\left|\left\langle 0\left|\sum_{j=1}^{3} \sigma_{z}^{j}\right| \Psi\right\rangle\right|^{2} \geq 0.005$ are shown. The different slopes are due to the fact that the qubits 1 and 2 were designed with $\alpha=0.5$ and $\alpha=0.475$ while the qubit 3 has $\alpha=0.45$. The lines shared by the qubits have the dimensions of $1.35 \mu \mathrm{m} \times 20 \mathrm{~nm} \times 100 \mathrm{~nm}$. Oxidation was done with 35 mTorr for $10 \mathrm{~min}$. We get $I_{\mathrm{c}, \max }^{\mathrm{SQUID}} \approx 5.4 \mu \mathrm{A}$.

ing the currents $\tilde{I}_{\mathrm{p} k}$. Based on the measured normal state resistivity of $\rho_{4.2 \mathrm{~K}}^{A l}=11 \mu \Omega \mathrm{cm}$ we estimate using 19 $L_{\mathrm{kin}}=\left(\Phi_{0} e R_{\mathrm{n}}\right) /\left(\pi^{2} \Delta_{\mathrm{BCS}}\right)$ that $M_{13}=M_{23}=31 \mathrm{pH}$. In order for this to be consistent with the coupling energy the shielding should be about $11 \%$. Due to the coupling we observe more than three levels and everywhere the spectrum lines cannot be simply associated with a particular qubit. Roughly speaking, the line having a minimum of about $2.5 \mathrm{GHz}$ corresponds to the qubit 3 and the other two minima around $2.2 \mathrm{GHz}$ correspond to the qubits 1 and 2 . This can be justified by noting that the qubit 3 is the only one coupled strongly to two other qubits. The slightly higher tunneling energy is because the qubit 3 was fabricated with $\alpha=0.475$ as 
the designed ratio of the large and small junction area while qubits 1 and 2 had $\alpha=0.5$. Since we are using only one control magnet we can write the flux biases as $\Phi_{j}=\Phi+\Delta \Phi_{j}$. The small offsets $\Delta \Phi_{j}$ are, in the absence of trapped vortices, due to slight differences in the areas. Since we only work in rather small range around $\Phi_{0} / 2$ the effect of area difference on the slope is not significant. We choose a convention where $\Delta \Phi_{3}=0$. Note however that due to the interaction between qubits, not even the qubit 3 has minimum exactly at $\Phi_{0} / 2$. The lack of visibility of some levels at certain field can be understood as small transition matrix element. In fact, in the theoretical calculation we have plotted only levels $|\Psi\rangle$ for which $\left|\left\langle 0\left|\sum_{j=1}^{3} \sigma_{z}^{j}\right| \Psi\right\rangle\right|^{2} \geq 0.002$.

Figures 3$]$ and 4 illustrate measured spectra for samples B and C. As can be seen the calculations again agree extremely well with the measurement. Together with sample A the samples cover a very wide range of parameters. It is especially noteworthy that the coupling energy can be made as large as $6 \mathrm{GHz}$ without using coupling junctions 9 . The shielding effect as well as the coupling inductance are expected to be very large in these samples since the line shared by the qubits and SQUID is made thinner and narrower than in sample A. A prediction based on normal state resistance yields the estimate $M_{13}=M_{23}=74 \mathrm{pH}$. In order to explain the observed coupling energy the shielding in sample B should be about $40 \%$ and in sample $\mathrm{C}$ about $14 \%$. These numbers are reasonable although hard to verify. The scaling of these percentages is correct as a function of $I_{\mathrm{c}, \max }^{\mathrm{SQUD}}$.

Even though the predicted kinetic inductances are large one may suspect that the large coupling could be partially due to shared Josephson inductance $L_{\mathrm{J}}$. Namely, a fraction of the persistent current of each qubit could flow through the large junctions of the SQUID. However, this effect may only reduce the coupling from the prediction based on shared kinetic inductance since the effect of the large shared $L_{\mathrm{J}}$ is cancelled by the fact that the fraction of the persistent currents flow- ing through the big junctions decreases with increasing $L_{\mathrm{J}}$. Explicitily, if the currents of two qubits $k$ and $l$ can flow through two inductances $2 L_{1}$ (kinetic) and $2 L_{2}$ (kinetic plus Josephson), and if half of these inductances are shared with the other qubit, then $J_{k l}=L_{1} L_{2} /\left(L_{1}+\right.$ $\left.L_{2}\right) I_{\mathrm{p} k} I_{\mathrm{p} l}$. This clearly is maximal for $L_{2} \rightarrow \infty$. An additional experimental point related to this is that in all samples we reproduced the measured spectrum well by setting the direct coupling of the qubits 1 and 2 to zero even though their currents have a chance to flow through a "shared" large Josephson junction.

In conclusion we have carried out spectroscopic measurements of three strongly coupled flux qubits. We demonstrated that one can achieve a wide range of coupling strengths using kinetic inductance for the coupling. The relatively high coupling energy is attributable to the rather large normal state resistivity of our aluminum film. For instance in Ref. 19 the resistivity seems to be about 7 times smaller while e.g. in Ref. 20 it is about half of our value. We find that the effective three-qubit Hamiltonian describes the measured spectrum well. Based on our experience it is possible to reproducibly fabricate qubits whose flux biases differ by less than $1 \%$ even when using only a single bias coil. The different effective area of qubit 3 in all samples is due to the fact that its position is not symmetric with respect to other qubits. However, it is possible to tune the area very accurately by adjusting the layout. The similar areas enable optimal point biasing of many qubits without strongly coupled independent bias lines. The experiments reported in this paper are a step towards the tunable coupling of flux qubits. The parameters of sample $\mathrm{C}$ in particular are very close to those required for the parametric coupling using a third qubit ${ }^{16}$. However, for good enough coherence the coupling to the readout will have to be modified to be more symmetric than in the present design.

We would like to thank M. Grajcar for useful discussions.

* Electronic address: niskanen@frl.cl.nec.co.jp

1 C. Rigetti, A. Blais, and M. Devoret, Phys. Rev. Lett. 94, 240502 (2005).

2 P. Bertet, C.J.P.M. Harmans, and J.E. Mooij, Phys. Rev. B 73, 064512 (2006).

3 M. Grajcar, Y.-X. Liu, F. Nori, and A.M. Zagoskin, e-print cond-mat/0605484

4 Yu.A. Pashkin et al., Nature (London) 421, 823 (2003).

5 T. Yamamoto, Yu.A. Pashkin, O. Astafiev, Y. Nakamura, and J.S. Tsai, Nature (London) 425, 941 (2003).

6 A.J. Berkley et al., Science 300, 1548 (2003).

7 J.B. Majer, F.G. Paauw, A.C.J. ter Haar, C.J.P.M. Harmans, and J.E. Mooij, Phys. Rev. Lett. 94, 090501 (2005).

8 R. McDermott et al., Science 307, (2005).

9 M. Grajcar et al., Phys. Rev. Lett. 96047006 (2006).

10 S.H.W. van der Ploeg et al., e-print cond-mat/0605588

11 M. Steffen et al., Science 313, 1423 (2006).

12 D. Vion et al., Science 296, 886 (2002).

13 P. Bertet et al., Phys. Rev. Lett. 95, 257002 (2005).

14 A. Wallraff et al., Phys. Rev. Lett. 95, 060501 (2005).

15 F. Yoshihara, K. Harrabi, A.O. Niskanen, Y. Nakamura, and J.S. Tsai, e-print cond-mat/0606481 [Phys. Rev. Lett.(to be published)].

16 A.O. Niskanen, Y. Nakamura, and J.S. Tsai, Phys. Rev. B 73, 094506 (2006).

17 J. Claudon, F. Balestro, F.W.J. Hekking, and O. Buisson, Phys. Rev. Lett. 93, 187003 (2004).

18 I. Chiorescu, Y. Nakamura, C.J.P.M. Harmans, and J.E. Mooij, Science 299, 1869 (2003).

19 A.C.J. ter Haar, Ph.D. Thesis, Delft University of Technology (2005).

${ }^{20}$ F. Balestro, J. Claudon, J.P. Pekola, and O. Buisson, Phys. 
Rev. Lett. 91, 158301 (2003). 